\title{
Clinical implications of cephalic vein morphometry in routine cardiac implantable electronic device insertion
}

\author{
R. Steckiewicz, E. Świętoń, P. Stolarz, M. Grabowski \\ Department of Cardiology, Medical University of Warsaw, Poland \\ [Received 23 December 2014; Accepted 15 April 2015]
}

Background: Morphometric parameters of the venous vasculature constitute an important aspect in successful cardiac implantable electronic device (CIED) insertion. The purpose of this study was to present morpho-anatomical variations of the cephalic vein (CV) and their effect on the course of CIED implantation procedures, based on the patients from our centre.

Materials and methods: We analysed contrast venography results obtained during first-time lead placement. Venography was indicated in the cases of problematic lead introduction with either the CV cutdown or axillary/subclavian vein puncture techniques. The 214 cases of venography (15\%) performed out of 1425 first-time lead placement in the period 2011-2013 were divided into 9 subgroups according to the most commonly observed CV variations of similar morpho-anatomical features that limited the use of the CV cutdown technique for lead insertion.

Results: The following CV morphometric parameters were found to be unfavourable in terms of lead placement: CV diameter of $\leq 1 \mathrm{~mm}(18 \%)$, sharp curvature of the terminal CV segment as it joined the axillary vein (14\%), terminal CV bifurcation (9\%), additional CV branches (7\%) or tributaries (7\%), stenoses (5\%), sharply winding course (5\%), single CV with a supraclavicular course (4\%).

Conclusions: The radiographic records obtained during the procedures allowed us to assess the prevalence of those atypical CV variations in our study group, with graphic presentation of characteristic types and sporadically reported CV variations. (Folia Morphol 2015; 74, 4: 458-464)

Key words: contrast venography, cephalic vein, transvenous lead placement

\section{INTRODUCTION}

Diagnostic and technological advances, along with the expanding indications for cardiac implantable electronic device (CIED) insertion have been steadily increasing the rate of procedures for pacemaker, implantable cardioverter-defibrillator (ICD), and cardiac resynchronisation therapy system implantation. The first stage of these procedures requires accessing the venous lumen successfully via cephalic vein (CV) cutdown, axillary vein (AV)/subclavian vein (SV) puncture, or a combination of both approaches [4].

The CV cutdown technique performed in the deltopectoral triangle is typically the first and preferred attempt at venous access, as it is associated with

Address for correspondence: R. Steckiewicz, MD, PhD, Department of Cardiology, Medical University of Warsaw, ul. Banacha 1A, 02-097 Warszawa, Poland, tel: +48 22599 2958, e-mail: r.steckiewicz@pro.onet.pl 


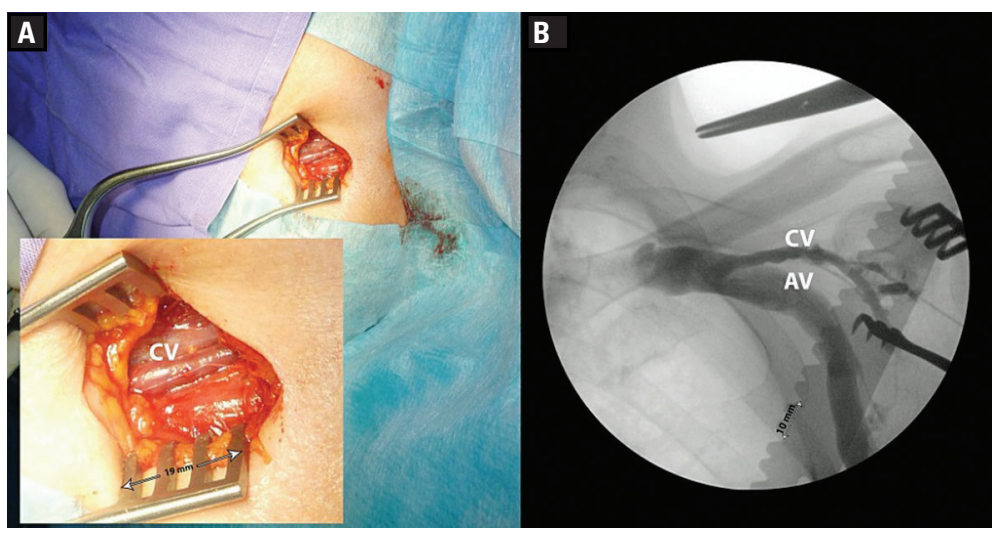

Figure 1. The methods and extent of cephalic vein (CV) morphology and anatomy assessments; A. Visual assessment: showing the site of lead insertion in the dissected fragment of the vessel; B. Radiographic assessment following contrast administration reveals further course of the CV (in the case presented here the CV-AV confluence angle was acute, pointing medially, which would facilitate the passage of the lead, also visible is a calibrated X-ray measuring ruler, Braun); AV — axillary vein.

a lower risk of traumatising the surrounding structures in comparison with the AV puncture approach $[3,9,10,15]$.

Lead introduction via the CV into the AV depends on favourable morphometric parameters of the former vessel. Visual inspection of the vessel is limited only to the segment exposed during the procedure, with further segments of the vessel visualised with contrast imaging $[5,11]$. Venography is still the only assessment that can be conducted during the procedure to help the operator decide whether or not to use the given vessel $[13,14]$.

The purpose of our study was to present interpersonal morphometric and anatomical CV variations based on intra-procedure venography assessments. The data collected during CIED implantation procedures allowed us to present rarely encountered venous configurations.

\section{MATERIALS AND METHODS}

Venograms conducted in the period from 2011 to 2013 during first-time CIED implantation in 214 patients (89 females and 125 males; mean age $72.3 \pm 10.9$ years) were included in the study. Contrast venography was used in 15\% of the 1,425 CIED procedures conducted in that period and requiring the insertion of 1 to 3 leads into the venous system.

The intended primary site of the procedure was the left subclavian region. The morphometric condition of the venous vessels allowed for this approach in 208 cases, whereas in 6 cases the procedure was conducted on the right side for the following reasons: venographic evidence of advanced post-thrombotic lesions in the left SV in 5 patients and a supraclavicular course of the CV in 1 patient.
CV morphometric parameters were assessed, with or without venography, with respect to whether or not the vessel would allow for the passage of at least one CIED lead. The same issue was investigated via contrast-enhanced venographic images of the CV in the deltopectoral triangle, i.e. between the site of lead insertion and the T-junction with the AV.

Venography was indicated in the cases requiring an assessment of venous vasculature anatomy: in cases of expected or already existing lead passage obstruction within the CV, AV or SV; to specify the exact point of AV and/or SV puncture while using a lead introducer kit.

The cardiac device/lead placement procedure was conducted according to the local protocol: a linear 4-6 cm (depending on the size of the implanted device) incision along the deltopectoral groove was made under local anaesthesia in the left infraclavicular region. This location of the entrance to the device pocket allowed for visual assessment of a longer segment of the vessel than if the incision had been parallel to the clavicle (Fig. 1A, B). The following parameters were assessed: CV diameter at the site of lead insertion and the distance from lead insertion site to the junction of the CV with the AV, i.e. the site where the AV continues into the SV, which is marked on X-ray images by the anterior clavicular border.

The flow of the contrast agent was visualised with the OEC 9900 Elite (GE) workstation, in anterior-posterior view. The digitally recorded snapshot fluoroscopy mode was used predominantly; however, dynamic contrast flow was recorded via cineangiographic pulse mode in selected cases. 


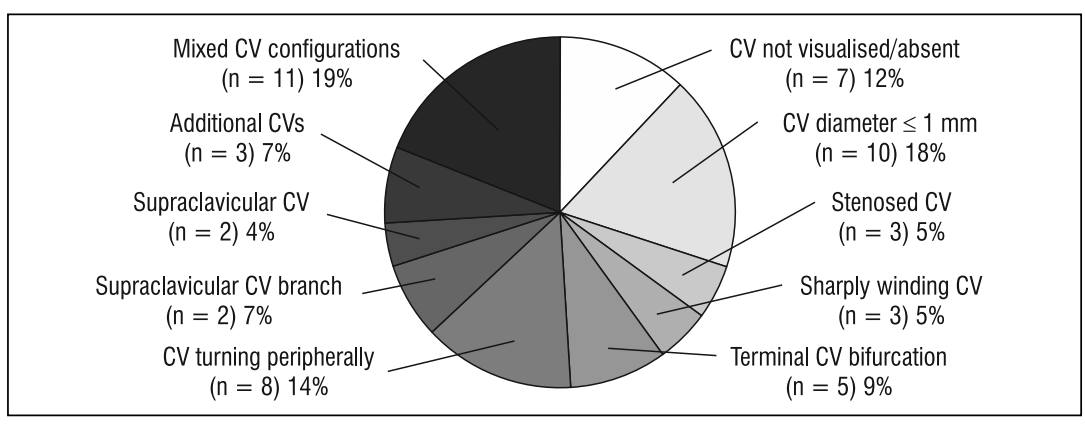

Figure 2. Morpho-anatomical cephalic vein (CV) stratification for the purpose of this paper.

Venography was conducted with contrast administration into superficial veins of the arm/forearm (conventional approach) or directly into the CV segment exposed via incision (selective approach). Selective venography images presented here are characterised by the absence of retrograde CV contrast filling peripherally to the administration site.

Based on the venography results, 9 morpho-anatomical subgroups of CVs were identified in the recorded radiographic images (Fig. 2-8). This presentation contains specially selected figures demonstrating subgroup-specific features.

\section{RESULTS}

The evaluated 214 procedures where venography was performed demonstrated the presence of the CV in 207 (96.7\%) cases. In 7 (3.3\%) no cases CV was found either via direct exposure through tissue dissection along the deltopectoral groove or via radiographic imaging following contrast administration.

In 133 (62\%) procedures with venography, the CV lay superficially, beneath the fascia covering the adipose tissue of the deltopectoral groove, in the remaining cases finding the vessel required deeper dissection.

In 157 (76\%) cases the structure and morphometric parameters of the CV allowed for the passage of at least one lead into further segments of the venous vascular system, without the need of a guide catheter. This CV subgroup was characterised by the lumen diameter measuring $\geq 2 \mathrm{~mm}$ in all places along the length of the vessel and the CV-AV confluence site at an angle that favoured further, proximal, progress of the lead (with the vertex of the acute angle pointing medially, Fig. 2B).

In 50 cases the morphometric parameters of the CV prevented lead introduction into further segments of the venous system, which - including 7 cases where the CV was not visualised - constituted $27 \%$ of all analysed procedures (Fig. 2).
In 6 out of 50 cases where the vessel's morphology and anatomy did not facilitate direct lead passage (12\%), successful lead placement was nevertheless achieved by using a guidewire and/or guide catheter from the lead introducer kit.

The diameter of the analysed vessel in the segment intended to convey the lead ranged from $<0.1 \mathrm{~cm}$ to $0.9 \mathrm{~cm}$ (mean $0.33 \pm 0.15 \mathrm{~cm}$ ), with values of $0.8-0.9 \mathrm{~cm}$ found only in 2 cases (Fig. 3A, B). The CV diameter of $\leq 0.1 \mathrm{~cm}$ practically excluded the introduction and passage of cardiac pacing leads into further segments of the venous vascular system in $20 \%$ of the cases presented here.

The distance to the CV-AV confluence, measured from the anterior clavicular border (beginning of the $\mathrm{SV}$ ) ranged from $0 \mathrm{~cm}$ to $9.0 \mathrm{~cm}$ (mean $2.4 \pm 2.4 \mathrm{~cm}$ ) (Fig. 4A, B).

In 11 cases the CV lumen failed to maintain constant diameter in various ways, including stenoses, sharp curves, and terminal bifurcations (Fig. 5A, B).

Segmental stenoses, either isolated or multiple, of the vascular lumen were found in 3 cases, with no change observed following lidocaine administration directly into the CV segment visualised in the device pocket. In 5 cases the CV termination was divided just distally to the CV-AV confluence site, with reduced diameter of both narrow branches precluding direct lead passage (Fig. 5B). In further 3 cases, the vessels had a sharply curved course.

In 8 cases, the CV arched and doubled back to empty into the $A V$ at an acute angle whose vertex pointed peripherally (Fig. 6A, B).

Our data showed 4 cases of the CV variation where the vessel gave off a branch coursing superiorly to the clavicle and emptying into the external jugular vein (EJV). In 2 of these cases the CV branching site was located within the exposed segment of the vessel (Fig. 7A). Additional CVs joining the main vessel in the deltopectoral triangle were found in 3 cases (Fig. 7B). 


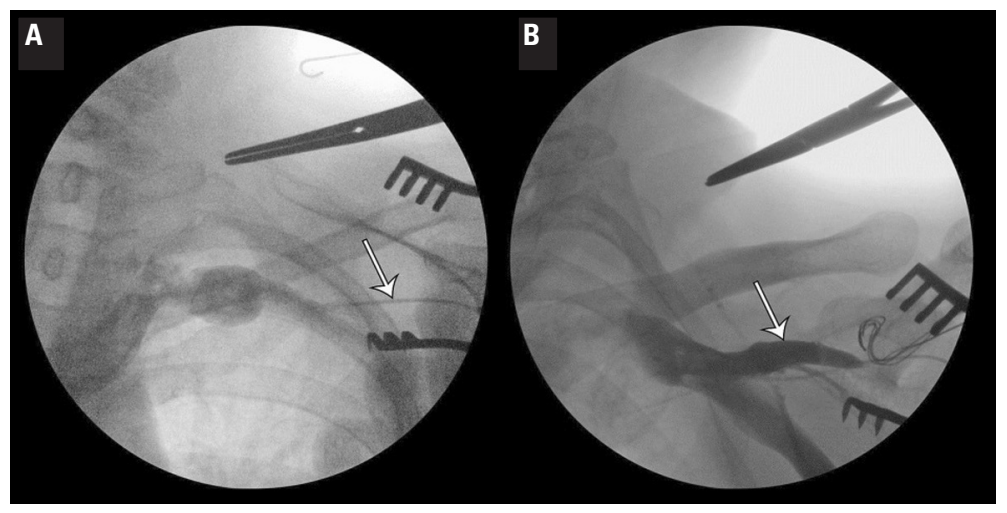

Figure 3. Cephalic vein (CV) diameter variations (white arrows); A. Narrow, poorly enhancing vessel following contrast administration; B. Wide vessel with the diameter similar to that of the axillary vein (in both cases selective venography was performed).

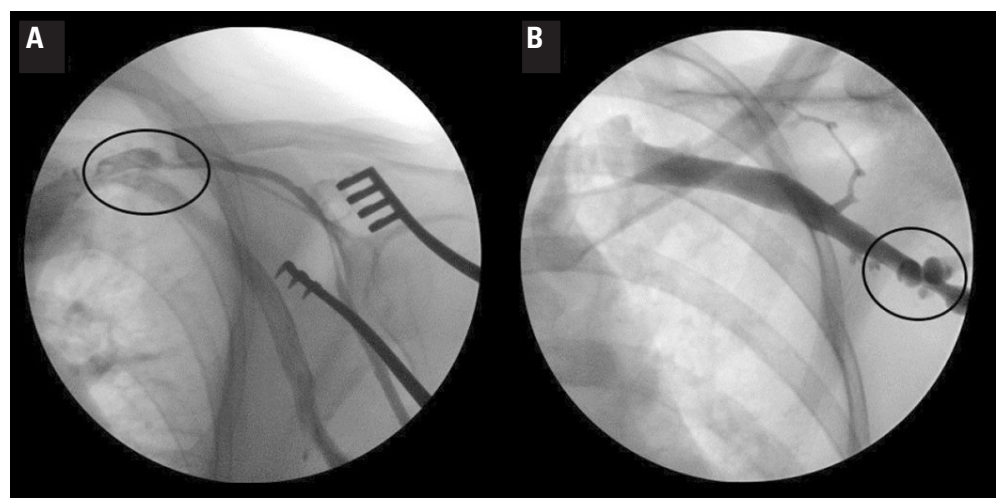

Figure 4. Cephalic vein-axillary vein (CV-AV) confluence site variations (black oval); $\mathbf{A}$. The CV outflow is located medially, close to the clavicle, the vessel is visible within the dissected pocket (B) the CV outflow is located peripherally, below the site where the vessel is typically searched for in the bed of the device. Direct inspection suggested a lack of the vessel, whose presence was detectable only following contrast administration (in both cases conventional venography was conducted).

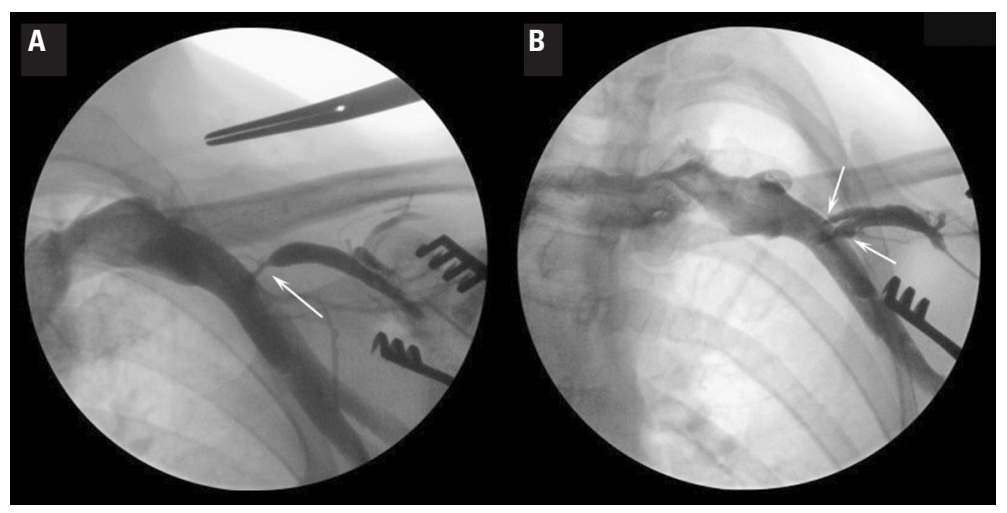

Figure 5. Segmental changes in cephalic vein morphology and lumen diameter (white arrows); A. Stenoses; B. Terminal bifurcation.

In 2 cases, the CV was found to be transposed superior to the clavicle, prior to emptying into the EJV (Fig. 8A, B).
Mixed CV configurations combined morphometric features similar to those from the individual subgroups, with varied degree of predominance. 


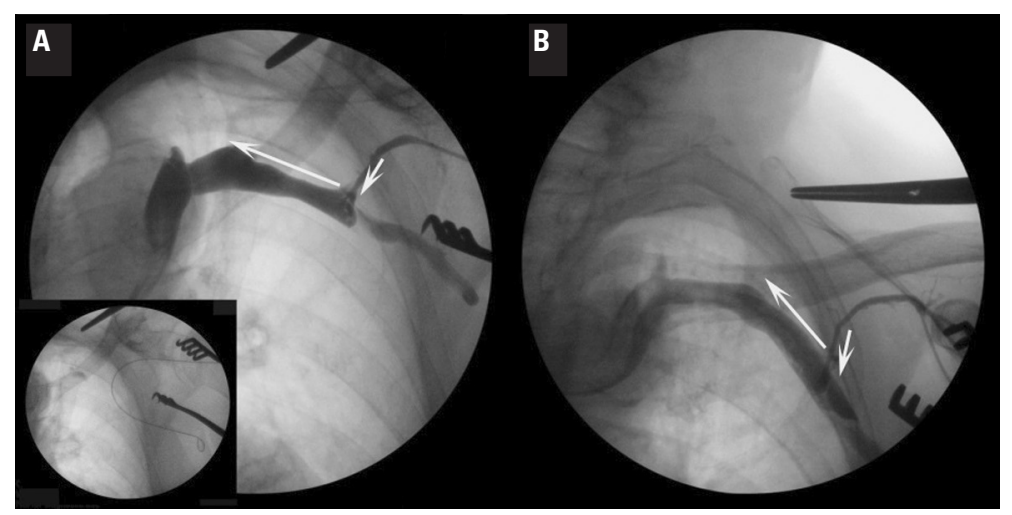

Figure 6. Cephalic vein (CV) (short arrow along the vessel) draining into the axillary vein (AV) (long arrow along the vessel); A. CV empties into the AV at an angle approximating 90 degrees, forming a "T-junction". The introduced lead is deflected peripherally; B. The CV with an arching retrograde acute angle at the junction with $\mathrm{AV}$, with the vertex pointing peripherally.

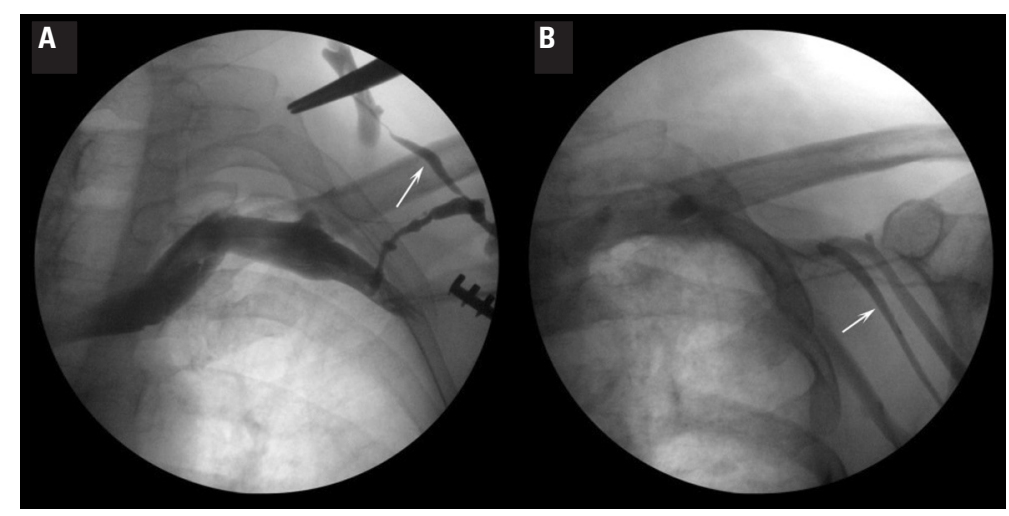

Figure 7. The cephalic vein (CV) with additional vessels (white arrows), visible in the device pocket; A. A branch of the CV that courses superiorly to the clavicle and empties into the external jugular vein; B. An additional CV joining the CV proper in the area of the CV-AV confluence; AV — axillary vein.

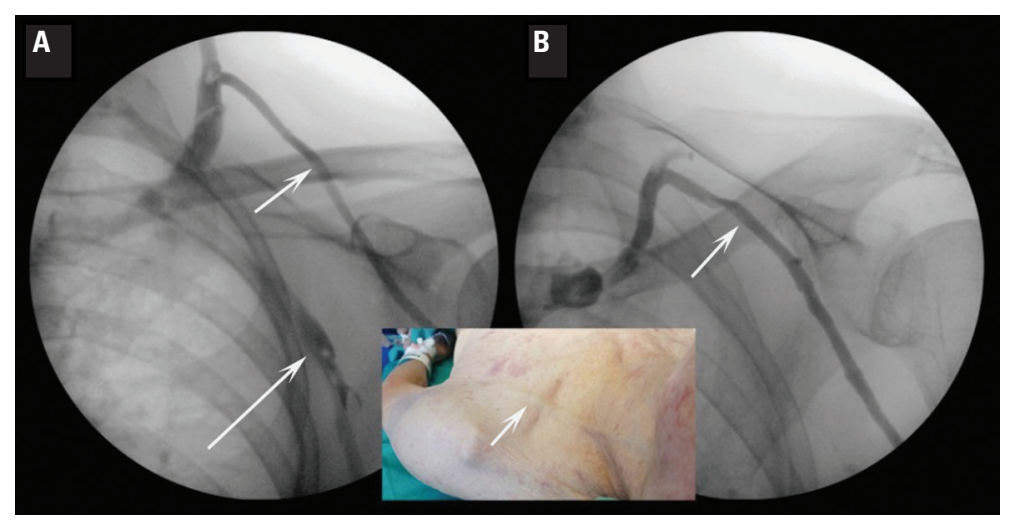

Figure 8. Both cases of a supraclavicular course of the cephalic vein (CV) (short arrow) and their drainage site into the external jugular vein; A. A patent axillary vein (AV) with normal, contrast filled lumen is also visible (long arrow); B. The AV fills poorly with contrast, whereas the CV is clearly the dominant vessel in the venous drainage of the left upper limb. The CV can be seen bulging through the skin along its supraclavicular course.

\section{DISCUSSION}

Effective introduction of continuous cardiac electrotherapy requires successful venous access into cardiac chambers [4]. The CV cutdown technique has no disadvantages of the AV/SV puncture approach, and is usually the primary choice of operators for introducing leads into the venous system $[9,15]$. Finally, the usefulness of the CV in terms of successful lead passage is determined by morpho-anatomical parameters of the vessel. A lack of optimal parameters 
has been associated with an estimated failure rate of $25-40 \%$ of cases, which is consistent with our findings (27\%) [2].

The rate of anatomical obstacles to passing the leads through the CV has been higher on the left side [13]. The preferred site for this procedure at our centre is the left subclavian region, which affected the scope of our analyses of CV anatomical variations.

Small vessel diameter $(\leq 0.1 \mathrm{~cm})$ was the reason for lead passage failure in $20 \%$ of cases (Fig. 3A). This factor has been reported as the cause of failure in approximately $17-20 \%$ of cases. However, the failure rate also depends on the number and structure of the leads introduced via the $C V$, vessel wall elasticity, operator's experience, etc. $[2,8,12,14]$. Moreover, we found no CVs with a diameter of over $1.0 \mathrm{~cm}$, with the mean values of CV diameter consequently lower [6]. Lead placement is easier in cases of larger CV lumen; however, a large CV lumen is also associated with a greater role of the vessel in venous drainage. Luminal occlusion in such cases can lead to clinical manifestations and venous blood flow obstruction, until collateral circulation develops. Our data included 2 cases of such CV occlusion. The vessels measured $0.8 \mathrm{~cm}$ and $0.9 \mathrm{~cm}$ in diameter (Fig. 3B).

We realise that the evaluated parameters, including CV diameters, are only relatively accurate. Our measurements were limited to the lead insertion site and showed a correlation with vascular wall tension (hydration), its mechanical irritation (constriction), venography, etc. These variables have no impact on vascular parameters in pathomorphological studies.

If CV contrast venography is not performed, CV may be left undetected, especially if the CV-AV confluence site is decidedly below than the area where it is typically expected. With such anatomical positioning of these veins the CV may seem to be absent, when in fact it is not (Fig. 4B). Such cases seem to be more common when the incision for device placement is made parallel to the clavicle. This approach provides a shorter exposed segment of the CV in comparison with the incisions made medially along the deltopectoral groove.

A direct passage of a lead through the CV was hindered in cases of lumen diameter variations in the form of segmental stenoses, winding course, and terminal bifurcation into 2 veins with smaller diameters (overriding type) (Fig. 5A, B). In 6 such cases, lead placement was possible only after initial guide catheter insertion, with the success of this technique also reported in other publications [8].
One of the frequently found CV variations (14\%) was a sharp curvature in the terminal segment causing the vessel to double back and join the AV at an acute angle pointing peripherally. This anatomical formation hindered lead manipulation to the extent that depended on the measure of the confluence angle, and in the case of an angle $<90^{\circ}$ made it practically impossible to steer the lead in the desired direction (Fig. 6A, B).

The presence of additional vessels joining or branching off the CV sometimes makes it difficult to distinguish between them and choose the right vessel during the procedure. In such cases, the success of the procedure depends on the order in which the vessels are exposed, the relative proportion of their diameters, and their spatial arrangement. The relevant noteworthy finding in the evaluated material was a CV with a branch traversing over the clavicle prior to its junction with the EJV (Fig. 7A) [7]. The presence of a vessel emptying into the $\mathrm{CV}$ proper in the deltopectoral triangle may pose a similar problem (Fig. 7B).

An operator who encounters an additional vessel, with morphology and course similar to those of the CV proper, in combination with conditions hindering lead manipulation may abandon his or her further search for the CV proper. Pathomorphological studies demonstrated the presence of 1 vessel joining the CV in $30 \%$ of cases, 2 vessels in $70 \%$ of cases [6]. In our study, the CV variation with 1 tributary was found in 3 patients; although admittedly, the evaluation included only the CV segment limited to the length of the deltopectoral groove.

During the study period, there were 2 cases where the $C V$ was found traversing over the clavicle and joining the EJV (Fig. 8A, B), which is a variety very rarely reported in the literature $[1,5,7]$. In 1 of those cases, recording venous flow through the AV failed despite 2 attempts at contrast enhancement (Fig. 8B). A venographic examination showed that the CV has taken over the venous drainage and became the dominant blood drainage vessel for the upper limb. In view of this, the device was eventually implanted on the right side, due to the fact that an occlusion of this CV following lead placement would severely disrupt venous blood flow within its drainage area.

\section{Limitations of the study}

This study is not without limitations. The quantitative distribution of the presented morpho-anatomical 
variations of the $\mathrm{CV}$ does not reflect their actual prevalence in the population. Our material came from a group of patients, pre-selected based on anticipated venous problems. We did not analyse correlations of the observed morphometric parameters of the CV vs. the specific construction and composition parameters of the lead models used.

In comparison with previous publications, our study - despite its limitations - seems to present a detailed portrayal of morpho-anatomical CV variations, including the variations found only in isolated literature reports, as well as addresses the impact of those variations on the course of cardiac pacing device implantation.

\section{CONCLUSIONS}

The degree of interpersonal variation in morphometric CV parameters in the vessel segment used for lead placement limits the use of the CV cutdown technique, despite novel modifications of the procedure (such as guide catheter use).

Supraclavicular course of the $\mathrm{CV}$ is a rare finding; however, if this anatomical variation is suspected during a physical examination, the patient should be qualified for pre-procedure contrast venography to visualise the morpho-anatomical configuration of veins found in the deltopectoral triangle.

\section{REFERENCES}

1. Kim D-I, Han S-H (2010) Venous variations in neck region: cephalic vein. IJAV, 3: 208-210.

2. Knight BP, Curlett K, Oral H, Pelosi F, Morady F, Strickberger SA (2002) Clinical predictors of successful cephalic vein access for implantation of endocardial leads. J Interv Card Electrophysiol, 7: 177-180.

3. Kolettis TM, Lysitsas DN, Apostolidis D, Baltogiannis GG, Sourla E, Michalis LK (2010) Improved 'cut-down' technique for transvenous pacemaker lead implantation. Europace, 12: 1282-1285. doi: org/10.1093/europace/euq173.

4. Lau EW (2007) Upper body venous access for transvenous lead placement-review of existent techniques. Pacing
Clin Electrophysiol, 30: 901-909. doi: 10.1111/j.1540-8159.2007.00779.x.

5. Lau EW, Liew R, Harris S (2007) An unusual case of the cephalic vein with a supraclavicular course. Pacing Clin Electrophysiol, 30: 719-20. doi: 10.1111/j.1540-8159.2007.00736.x

6. Loukas M, Myers CS, Wartmann ChT, Tubbs RS, Judge T, Curry B, Jordan R (2008) The clinical anatomy of the cephalic vein in the deltopectoral triangle. Folia Morphol, 67: 72-77.

7. Lum C, Ladenheim ED (2013) An interesting clinical case: variant of the cephalic vein emptying into the internal jugular vein. Semin Dial, 26:E11-2. doi: 10.1111/j.1525-139X.2012.01102.x.

8. Neri R, Cesario AS, Baragli D, Monti F, Danisi N, Glaciale G, Gambelli G (2003) Permanent pacing lead insertion through the cephalic vein using an hydrophilic guidewire. Pacing Clin Electrophysiol, 26: 2313-2314. doi: 10.1111/j.1540-8159.2003.00365.x.

9. Parsonnet V, Roelke M (1999) The cephalic vein cutdown versus subclavian puncture for pacemaker/ICD lead implantation. PACE, 22: 695-697.

10. Povoski SP (2000) A prospective analysis of the cephalic vein cutdown approach for chronic indwelling central venous access in 100 consecutive cancer patients. Ann Surg Oncol, 7: 496-502.

11. Saaid A, Drysdale I (2008) Unusual termination of the cephalic vein. Clin Anat, 21: 786-787. doi: 10.1002/ca.20661.

12. Tobin K, Stewart J, Westveer D, Frumin H (2000) Acute complications of permanent pacemaker implantation: their financial implication and relation to volume and operator experience. Am J Cardiol, 85: 774-776, A9. doi:org/10.1016/S0002-9149(99)00861-9.

13. Tokano T, Nakazato Y, Shiozawa T, Konishi H, Hiki M, Kato Y, Komatsu S, Yamase M, Komatsu K, Hayashi H, Sekita G, Suwa S, Bito F, Kizu K, Sumiyoshi M, Daida H (2013) Variations in cephalic vein venography for device implantation-Relationship to success rate of lead implantation. J Arrhyth, 29: 9-12. doi: org/10.1016/j.joa.2012.05.009.

14. Tse HF, Lau CP, Leung SK (2001) A cephalic vein cutdown and venography technique to facilitate pacemaker and defibrillator lead implantation. Pacing Clin Electrophysiol, 24: 469-473.

15. Ussen B, Dhillon PS, Anderson L, Beeton I, Hickman M, Gallagher MM (2011) Safety and feasibility of cephalic venous access for cardiac resynchronization device implantation. Pacing Clin Electrophysiol, 34: 365-369. doi: 10.1111/j.1540-8159.2010.02975.x. 